Revista de Psicologia de la Salud, 1989, Volumen 1, № 1, Pág. 105 a 125

\title{
INVESTIGACIÓN DEL DOLOR EN LABORATORIO
}

\author{
Tomás Blasco Blasco y Ramón Bayés Sopena
}

Universidad Autónoma de Barcelona

\section{RESUMEN}

Se presenta una revisión de los procedimientos y estrategias que se utilizan para la investigación del dolor en condiciones de laboratorio, llevándose a cabo un análisis crítico de las ventajas, inconvenientes y limitaciones de los mismos. Finalmente, se apuntan algunas consideraciones susceptibles de constituir pautas para el desarrollo de futuras líneas de investigación.

\section{ABSTRACT}

A review on procedures and strategies used to investigate pain in lab conditions emphisizing a critical analysis on its advantages, difficulties and limits is focused here. Also guidelines are looked upon for future research.

\section{INTRODUCCIÓN}

El dolor es una experiencia compleja que presenta paradojas sorprendentes: por ejemplo, ante un mismo tipo de lesión hay individuos que no manifiestan sentir dolor, mientras que otros necesitan analgésicos; pero también se produce el fenómeno contrario, cual es que en muchas ocasiones no se encuentra una causa física que permita enjuiciar las manifestaciones de dolor del individuo, to que constituye un grave problema cuando este dolor tiene una duración prolongada y los recursos analgésicos tradicionales fracasan.

A pesar de que incluso los mismos profesionales de la Medicina han aceptado desde hace mucho tiempo que el dolor, además de un componente biológico, posee una dimensión psicológica, ha sido a partir de los problemas que ha planteado el dolor crónico que la psicología se ha interesado en el tema y ha intentado encontrar métodos y terapias que permitan al enfermo con dolor reducir o incluso eliminar sus molestias.

La investigación en este terreno se ha desarrollado fundamentalmente en dos vertientes: por un lado, la aplicación de diversos tipos de terapias a pacientes con dolor y, por otro, el estudio del dolor en condiciones de laboratorio, provocando una experiencia dolorosa en sujetos sanos. La importancia de este segundo tipo de estudios radica no sólo 
en la evaluación de terapias farmacológicas y no farmacológicas contra el dolor, sino también en la posibilidad de proporcionar un conocimiento de los factores que determinan la experiencia de dolor. Por lo tanto, el dolor experimental no es sino un intento de aproximarse al dolor clinico mediante una situación de laboratorio en la que se intentan evaluar algunos parámetros de la experiencia dolorosa.

Hay, sin embargo, un problema implícito al estudio del dolor en laboratorio y es la propia distinción entre dolor clínico y dolor experimental: la cualidad de ambos no es la misma. Si el dolor experimental fuera susceptible de equipararse en su totalidad -cualitativa y cuantitativamente- al dolor clínico, no hablaríamos de dolor clínico y dolor experimental, sino más bien de "dolor natural" y "dolor producido en el laboratorio". Por el contrario, el dolor clínico difiere del experimental en que es más agudo, más prolongado y menos predictible en su duración; menos sometido a finalización voluntaria $y$, normalmente, más productor de ansiedad. Por ello algunos autores consideran que las conclusiones sobre la acción de analgésicos en el dolor experimental no pueden extrapolarse directamente al dolor clínico. Así, por ejemplo, la morfina, que es muy eficaz en el tratamiento del dolor de origen patológico no lo es tanto en el tratamiento del dolor experimental; se ha especulado que esto podría deberse al componente de ansiedad que se encuentra en el dolor clínico pero no en el dolor experimental. Algo similar ocurre en el caso de los placebos, que son efectivos en un $35 \%$ de los casos clínicos y sólo un 5\% en el dolor experimental (Beecher, 1975; Miró, 1983).

A pesar de las limitaciones que en su extrapolación a la situación fuera de laboratorio presenta el dolor experimental y de los problemas intrínsecos que plantea, tanto en lo que refiere a procedimientos como en lo que refiere a medición, no cabe duda de que el trabajo en condiciones de laboratorio es necesario tanto para el conocimiento de los mecanismos fisiológicos y psicológicos que intervienen en el proceso de percepción del dolor, como para la evaluación de la efectividad de fármacos y otros procedimientos terapéuticos y que, por tanto, debería potenciarse la investigación en ese campo.

Consideraremos, pues, que el dolor experimental es aquel en que el estímulo que lo produce es conocido, manipulado y controlado por un investigador $y$ en el que los sujetos que lo padecen lo han aceptado voluntariamente. Por contra, el dolor clínico es aquel en que el estímulo que lo origina es, en muchos casos, desconocido, y no es manipulable ni controlable de una manera precisa en lo que refiere a su intensidad, duración y extensión, ni predecible en cuanto al momento de su aparición. 


\section{ASPECTOS GENERALES}

Aun cuando puedan concebirse métodos muy diversos para la producción y el estudio del dolor en el laboratorio, es obvio que no todos pueden ser igualmente útiles, ya que la precisión de las medidas a obtener variará de unos a otros, así como la posibilidad de que estas puedan ser reproducidas. Revés (1983) ha recopilado las condiciones exigidas por diversos autores para que un procedimiento sea válido para la medida del dolor y de las que destacaríamos las siguientes:

1. Los estímulos han de inducir una sensación de dolor claramente definida.

2. Los estímulos han de estar definidos claramente en términos físicos y han de poder ser cuantificables y reproductibles.

3. El estímulo no ha de causar daños irreversibles en el tejido.

4. Ha de ser sensible a la acción de los analgésicos.

Como veremos, no todos los procedimientos que actualmente se utilizan en la investigación de laboratorio cumplen todos y cada uno de estos requisitos, pero no por ello deben ser excluidos, ya que aportan, en mayor o menor medida, posibilidades de obtener información sobre los procesos del dolor.

La investigación de dolor en laboratorio se realiza en mamíferos inferiores y humanos. Puesto que el objetivo de nuestro trabajo es la evaluación del dolòr en humanos, daremos sólo unas pequeñas indicacionés de cómo se llevan a cabo las investigaciones en mamíferos inferiores y qué parámetros utilizan.

En primer lugar, debemos justificar el porqué de la investigación con animales. De acuerdo con Chapman, Casey, Dubner, Foley, Gracely y Reading (1985), hay dos razones fundamentales:

1. La experimentación animal permite la manipulación de variables experimentales que pueden conducir a una comprensión de los mecanismos del dolor y terapias analgésicas en niveles celulares y subcelulares.

2. Pueden servir como modelo de ciertas condiciones patológicas humanas en un ambiente controlado, en el que la precisión en la manipulación de variables fisiológicas o farmacológicas no es posible o éticamente justificable en humanos.

Por otra parte, no es solamente en los estudios sobre dolor en los que adquiere importancia la investigación animal en sus posibilidades de extrapolación al campo humano; baste recordar que cualquier investigación farmacológica se basa en una batería de pruebas que se aplica a muestras de animales -generalmente, ratas o ratones- y de cuyos resultados dependerá la utilización del fármaco en el ámbito humano.

Es evidente que si el estudio del dolor constituye algo complejo y difícil en el campo humano, en el que nos podemos servir del informe 
verbal, aún es más problemático el análisis de esta reacción cuando se trabaja con animales. De ahí que se utilicen medidas conductuales como inferencia sobre el dolor, las cuales son de dos tipos: conductas reflejas e indicadores de reacciones voluntarias.

En lo que se refiere a conductas reflejas, se utilizan las medidas de latencia de respuesta, como por ejemplo en el reflejo de levantar la cola -tail flick reflex-, reacción de encogimiento-salto -flinch-jump- o retirada de la pata -limb withdrawal-. Las limitaciones de estos métodos radican, por una parte, en el hecho de que la sensación de dolor no puede inferirse sólo de las medidas del reflejo, y que las respuestas espinales reflejas no miden las funciones superiores del Sistema Nervioso Central involucradas en la experiencia del dolor; por otra parte, la mayoría de los reflejos medidos en animales no se encuentran en el hombre y es por ello criticable la extrapolación o validez de los resultados a la especie humana.

Los indicadores de actividad voluntaria constituyen respuestas del animal a situaciones más complejas en las que suelen estar involucrados varios parámetros conductuales: conducta de retorcimiento del animal tras una inyección peritoneal -algunos autores consideran esta respuesta como un modelo del dolor visceral en humanos-, respuestas de vocalización que podrían reflejar el componente afectivo de la respuesta de dolor -aunque los estímulos no nocivos también pueden elicitar respuestas vocales-, conductas de escape y evitación, paradigmas de elección motivacional -el animal debe resistir una intensidad dolorosa antes de obtener una recompensa- o bien disminución de conductas habituales producidas por el dolor.

En conclusión, aun cuando la información que pueda obtenerse de estas investigaciones sea útil, no hay que olvidar que ésta abarca solamente el aspecto sensoriodiscriminativo de la experiencia dolorosa, proporcionando escasa información sobre el aspecto aversivo o emocional, parámetros cuya importancia en la reacción de dolor no debe olvidarse. Debe evitarse, pues, el realizar generalizaciones inadecuadas sobre el dolor a partir de los resultados obtenidos en mamíferos inferiores, aunque ello no sea obstáculo para presuponer que fenómenos que se detectan en especies inferiores, como el caso de la analgesia inducida por estrés (Lewis, Canon y Liebeskind, 1980; Maier, Drugan y Grau, 1982) no sean susceptibles de producirse también en humanos (Willen, Dehen y Cambier, 1981), como veremos más adelante.

Centrándonos ya en la investigación sobre sujetos humanos, dirigiremos nuestra atención a los diversos tipos de dolor experimental, que clasificaremos en dos grupos en función de la clase de sensación que producen en el individuo, entendiendo esta como fásica o tónica. En el primer caso, tendríamos aquellos procedimientos que dan lugar a varias 
estimulaciones de duración breve -normalmente, escasos segundoscada una y que son los habitualmente utilizados en el estudio de los umbrales de dolor; en el segundo caso, aquellos en los que se usa una estimulación ininterrumpida más prolongada -de más de diez o quince segundos-.

Pero debemos comenzar haciendo un breve repaso a la terminología utilizada en la literatura y que consideramos poco clara, ya que con los mismos términos se refieren procesos diferentes o bien se utilizan varios nombres para una misma medida, lo que da lugar a una confusión considerable a la hora de comparar los resultados.

No parecen existir desacuerdos en cuanto al concepto de umbral de dolor, entendiendo éste como aquella magnitud de estímulo -intensidad, duración, extensión- para la cual el sujeto indica que está experimentando una sensación que identifica como dolor. Hablamos de magnitud de estímulo ya que, dependiendo del procedimiento experimental utilizado, se registra una u otra dimensión de éste. Así, en el caso del dolor fásico, -ya sea utilizando descargas eléctricas, radiación calórica o presión en los dedos o en la piel- el umbral del dolor se refiere a la intensidad de estímulo -miliamperios, grados centígrados, etc.-; en cambio, en el caso del dolor tónico -agua fría, isquemia- el umbral de dolor se mide por el tiempo que ha pasado desde que se aplicó el estímulo al sujeto hasta que éste indica que comienza a sentir dolor ya que, teóricamente, la intensidad de estímulo aplicado ha permanecido constante.

Nos parece conveniente denominar a esta magnitud umbral inferior del dolor y lo definiremos como aquel valor de estímulo ante el cual el sujeto comienza a percibir la experiencia como dolorosa. Por lo tanto, y dependiendo de si el procedimiento es fásico o tónico y del tipo de estimulación utilizada -descarga eléctrica, agua fría, etc.- el umbral inferior de dolor se registrará en unas $u$ otras unidades, aunque siempre con referencia al mismo proceso.

La situación cambia completamente cuando nos referimos al término tolerancia. Algunos autores califican como tolerancia la máxima intensitensidad de estímulo que un sujeto puede soportar antes de abandonar el experimento, o aquella que el sujeto directamente califica como intolerable o que le lleva a realizar conductas de huida ante el estímulo -por ejemplo, retirar la mano del agua en el procedimiento del agua fría-. Otros se refieren con ello a la diferencia existente entre el umbral inferior de dolor y la máxima estimulación dolorosa que el sujeto es capaz de soportar antes de calificarla como intolerable. En nuestra opinión, el término tolerancia conlleva una denotación ambigua: ¿es lo mismo "tolerar" una intensidad de estímulo elevada durante poco tiempo que "tolerar" una estimulación menos intensa pero durante un lapso de tiempo mucho más prolongado?. ¿En qué caso se "tolera" mejor el dolor?. Mu- 
chas veces, el objetivo terapeútico es conseguir que el dolor pase de intolerable a tolerable y ello puede significar muchas cosas: no sólo que el individuo soporte más intensidad, sino que la soporte mejor, que no interrumpa su actividad normal. Creemos que la palabra "tolerancia" se refiere a un concepto pluridimensional que desborda el aspecto unidimensional -la intensidad- de la experiencia de laboratorio. Por nuestra parte, propondríamos la utilización de los términos que mencionamos a continuación.

Aquel valor de magnitud de estímulo para el cual el sujeto califica la experiencia de dolor como intolerable $\mathrm{y} / \mathrm{o}$ le conduce a realizar conductas de huida ante éste lo denominaríamos umbral de retirada y lo registraríamos en las mismas unidades que el umbral inferior de dolor. Por lo tanto, no diremos que un sujeto tiene mucha o poca tolerancia al dolor, sino que tiene un umbral de retirada alto o bajo con relación al tipo de producción de dolor que se está utilizando. Es obvio que tanto el umbral inferior de dolor como el umbral de retirada son magnitudes que sólo pueden determinarse en condiciones de laboratorio.

La diferencia existente entre el umbral inferior de dolor y el umbral de retirada y que, como ya hemos dicho, algunos autores denominan tolerancia, creemos debe sustituirse por el término dominio de sensibilidad al dolor, tal como ha sido propuesto por Wolff (1983). Ciñéndonos a la diferencia aritmética entre umbral de retirada y umbral inferior de dolor para definir el dominio de sensibilidad al dolor, diremos que un individuo tiene más o menos dominio de sensibilidad al dolor. Esto es algo muy importante a la hora de evaluar la eficacia de una terapia o un analgésico, ya que el dominio de sensibilidad al dolor parece correlacionar con el concepto clínico "capacidad de aguantar el dolor" y sería una característica propia de cada individuo (Wolff, 1983).

Concluiremos, pues, diciendo que para la medida del dolor experimental necesitamos conocer tres parámetros básicos: el umbral inferior del dolor, el umbral de retirada y el dominio de sensibilidad al dolor.

Otros parámetros pueden utilizarse en investigaciones concretas en función de los procedimientos utilizados, y considerando o no los informes subjetivos de dolor que proporciona el individuo; pero, en unestra opinión, estas tres magnitudes absolutas son las que siempre deberían tenerse en cuenta a la hora de evaluar la eficacia de las terapias y hacer comparaciones entre ellas.

Hemos hablado hasta ahora únicamente de "cantidad" de dolor y no de "cualidad". No negamos que el dolor experimental tenga unas cualidades determinadas, pero creemos que, en principio, éste es un aspecto más secundario. Obviamente, el conocer la cualidad del dolor es interesante en clínica para el diagnóstico diferencial, pero no creemos que sea necesaria esta taxonomía en el dolor experimental, aunque sí 
debamos conocer a qué se asemejan los diversos tipos de dolor experimental que podamos obtener, como veremos en el apartado 5. Cabe preguntarse si es separable la cualidad del dolor de la cantidad. Toda reducción de un fenómeno a algunos de sus componentes implica una pérdida de información y un alejamiento de la realidad, pero si hay algo que podemos controlar en el dolor experimental son los parámetros de intensidad, $y$, por tanto, es ahi donde, a nuestro juicio, debemos dirigir nuestros esfuerzos: a discernir lo más claramente posible cuáles son los "mecanismos" de la intensidad.

\section{PROCEDIMIENTOS}

En la investigación del dolor experimental se han utilizado procedimientos muy diversos. Nosotros los clasificaremos a partir de la taxonomía que ya hemos indicado; esto es, procedimientos de dolor fásico y procedimientos de dolor tónico. Aún cuando algunos procedimientos pueden generar ambos tipos de dolor, generalmente se sitúan dentro de uno de estos dos grandes apartados y así los consideraremos.

\subsection{Procedimientos de dolor fásico}

Son los utilizados tradicionalmente para obtener el umbral inferior de dolor y su aplicación se rige por los procedimientos clásicos de la psicofísica; es decir, administrando al sujeto el mismo estímulo en diversas ocasiones y variándolo en intensidad hasta que se determina el umbral inferior de dolor. De la misma manera puede hallarse el umbral de retirada.

Los métodos mecánicos de producción de dolor fueron los primeros en utilizarse y se han empleado frecuentemente, ya que suelen ser simples en su aplicación y aceptados sin problemas por la mayoría de los sujetos. Consisten en la aplicación de una fuerza que se genera mediante la contracción de un muelle sobre una superficie ósea del cuerpo como pueden ser los dedos (Spanos, Hodgins, Stam y Gwynn, 1984), sobre puntos sensibles de la piel como pueden ser los del abdomen (Patkin, 1975), o bien haciendo que los músculos del brazo venzan la resistencia de ese muelle (Rodríguez, 1983). El umbral inferior de dolor y el umbral de retirada se miden en unidades de fuerza. Esta técnica es también conocida como "técnica del algómetro" y los diversos autores la utilizan con más o menos modificaciones.

En lo que se refiere a la producción térmica de dolor fásico, se ha utilizado generalmente el calor como estímulo generador del dolor. La técnica consiste en enfocar la luz de una lámpara potente a una peque- 
ña zona de la piel del individuo que previamente ha sido teñida con tinta negra para asegurar la absorción total de la radiación por la piel. El umbral inferior de dolor y el umbral de retirada vendrían definidos en calorías. En los años 50, Hardy y otros investigadores utilizaron este método para preparar una escala de dolor, observando que había 21 diferencias de intensidad de dolor discriminables por el sujeto entre el umbral inferior de dolor y el umbral de retirada y crearon la unidad llamada "dol" que equivalía a dos de estas diferencias discriminables por el sujeto. Así pues, el intervalo entre el umbral inferior del dolor y el umbral de retirada sería de 10 dols. Sin embargo, este método ha estado sujeto a críticas diversas: hay una falta de control de las variaciones de la intensidad de color de la piel teñida $y$, además, pueden producirse cambios en la respuesta del sujeto como consecuencia de la estimulación repetida, aparte de existir un riesgo considerable de producir lesiones en el tejido. Por otra parte, la escala "dol" propueta por Hardy y colaboradores ha encontrado dificultades de replicación en muchos casos, habiendo quedado prácticamente fuera de uso en la actualidad.

En lo que refiere a la utilización del frío, Fruhstorfer y Lindblom (1983) parten del supuesto de que existen nociceptores específicos situados en los tejidos venosos, los cuales serían los responsables de la sensación de dolor cuando se aplica una estimulación fría sobre la piel. Para comprobarlo, produjeron isquemia en una vena de la mano de los sujetos en la que, al quedar vacía de sangre, inyectaron solución salina a diversas temperaturas. Sin embargo, la duración de esta estimulación dolorosa es algo que no queda controlado. Por su parte, Chery-Croze (1983) aplica estimulación fría directamente sobre la piel, encontrando una relación directa entre la intensidad de estímulo y la de dolor señalada por el sujeto.

Junto con las técnicas de calor radiante, las de estimulación eléctrica han sido también muy utilizadas. Ello es debido a la facilidad para controlar el estímulo eléctrico, cuantificarlo y aplicarlo. Normalmente, la descarga eléctrica se aplica sobre la piel o sobre la encía. Esta última se utiliza porque está menos expuesta a influencias externas como la temperatura o la sudoración y se ha utilizado a menudo para evaluar la potencia analgésica de un fármaco (Dworkin, Chen, Schubert y Clark, 1984). La descarga eléctrica en la piel se ha utilizado ampliamente en la investigación de laboratorio, aunque no siempre se ha aplicado de forma correcta, ya que la intensidad eléctrica que llega al individuo dependerá de la resistencia eléctrica de la piel, la cual varía según la activación del Sistema Nervioso Autónomo del individuo. Pero esto puede solventarse mediante las oportunas manipulaciones técnicas y el procedimiento adquiere un aumento de fiabilidad considerable. Recientemente, 
Bromm y Meier (1984) han utilizado una estrategia más sofisticada de estimulación eléctrica en la que el electrodo se halla situado bajo la piel, lo que reduce considerablemente la variabilidad de resistencia de la misma y produce una clara sensación de dolor calificada de punzante, aguda, molesta y cálida. La fiabilidad de las medidas intra e intersesiones es elevada y su capacidad evaluativa del potencial analgésico de narcóticos opiáceos -como método de validación del procedimiento- da resultados satisfactorios.

Los métodos químicos son los menos utilizados debido a que son más complejos en su aplicación y, además, al administrarse mediante una inyección tienden a producir una reacción inflamatoria en el tejido estimulado. Usualmente se utilizan soluciones de iodo, potasio, quinina o naloxona y las medidas se toman en términos de concentración de la solución o del tiempo que tarda una concentración determinada en producir dolor. Por otra parte, plantea el problema de que su duración y extensión son difíciles de controlar, lo que obstaculiza su replicabilidad. A pesar de ello, algunas investigaciones apoyan la utilización del método de inyección salina a diversas concentraciones y diversos niveles de presión como un procedimiento satisfactorio para la medida psicofísica del umbral inferior de dolor somático profundo, aunque reconocen que no es útil para la determinación de otras medidas (Wolff y Jarvik, 1975).

\subsection{Procedimientos de dolor crónico}

Conocemos dos procedimientos para la producción de dolor tónico: la isquemia y la inmersión de la mano en el agua fría (Cold Pressor Test).

El procedimiento de isquemia, también llamado oclusión vascular, consiste en privar de sangre a los músculos del brazo -mediante la utilización de un manguito o torniquete- y, por lo tanto, de oxígeno, y provocar la contracción de los mismos haciendo que el sujeto apriete rítmicamente con las manos una pelota de goma. El dolor se mide por la cantidad de trabajo realizado por estos músculos o por el tiempo que tarda el sujeto en manifestar que percibe una sensación de dolor. Se ha argumentado que el dolor producido mediante isquemia es mucho más similar al dolor clínico que cualquier otro procedimiento de los utilizados en laboratorio; sin embargo, plantea muchos problemas metodológicos en su utilización como dolor experimental (Wolff, 1978):

a) Es bastante poco fiable para la medición psicofísica del dolor: en ocasiones, el dolor aparece de manera brusca, aumentando la intensidad rápidamente hasta alcanzar el umbral de retirada. 
b) La aplicación del manguito o torniquete por sí misma produce en muchos sujetos una molestia mayor que la del dolor isquémico, mostrándose reacios a su aplicación

c) Finalmente, la fatiga producida por el ejercicio muscular precede a la aplicación del dolor real.

En cualquier caso, se requieren de 8 a 25 minutos -según los sujetos- para alcanzar el umbral inferior de dolor -otros autores indican que el dolor puede producirse antes: al cabo de 3 ó 4 minutos (Weisenberg, 1977)- . Por último, no pueden tomarse varias medidas en la misma sesión, ya que después de la primera prueba subsisten en el individuo una pequeñas molestias, producto de la presión del manguito, que permanecen durante varias horas. Todo ello hace que el procedimiento de isquemia sea muy poco usado en las investigaciones de laboratorio, aunque ocasionalmente se utilice conjuntamente con otras técnicas (García de Jalón, Harrison, Johnson, Kozma y Schnelle, 1985) para evaluar la potencia de un analgésico, a pesar de que la fiabilidad de los resultados que se obtienen está aún en discusión (Weisenberg, 1977).

El Cold Pressor Test es quizá el procedimiento que más se ha utilizado para evaluar la potencia analgésica de técnicas psicológicas y. farmacológicas, puesto que es el más sencillo en su utilización y el que produce menos ansiedad en los sujetos si se les compara, por ejemplo, con la estimulación eléctrica (De Blas y Labrador, 1984). Por otra parte, el Cold Pressor Test es una experiencia mucho más intensa en todas sus dimensiones que la descarga eléctrica. La causa de esto se halla en que produce patrones temporales de dolor diferentes a los otros métodos: el Cold Pressor Test produce dolor constantemente y hace que la intensidad del mismo aumente lentamente, mientras que la estimulación eléctrica se presenta con breves períodos de tiempo separados por largos espacios de ausencia de estímulo y, por lo tanto, de dolor. Coincidimos en esto con la opinión de Klepac, Dowling y Hauge (1981). Por otra parte, presenta ventajas similares frente al dolor isquémico ya que es más sencillo de aplicar que éste, produce menos ansiedad o temor en los sujetos y permite que el dolor aparezca en un tiempo más breve, aumentando su intensidad de manera más lenta. En todo caso, y según la temperatura del agua, suelen bastar dos o tres minutos de estimulación para alcanzar el umbral de retirada.

El procedimiento consiste, básicamente, en mantener un baño de agua fría a temperatura constante entre 0 y 2 grados centígrados -algunos autores utilizan temperaturas de 5 a 7 grados centígrados- en el que se sumerge la mano del individuo. A los pocos segundos, comienza a aparecer una sensación que el sujeto identifica claramente comodolor y que va aumentando paulatinamente de intensidad, hasta alcanzar el umbral de retirada en dos minutos, o menos, en la mayoría de 
los sujetos. Gracias a su sencillez de aplicación, ha sido utilizada por muchos autores para evaluar todo tipo de terapias contra el dolor: psicológicas (Ahles, Blanchard y Leventhal, 1983; Avia, 1980; Blitz y Dinnerstein, 1975; De Blas y Labrador, 1984; Gilligan, Ascher, Wolper y Bochachevsky, 1984; Hayes y Wolf, 1984; Hilgard, Ruch, Lange, Lenox, Morgan y Sachs, 1974; Linton y Gotestam, 1983; Pancyr y Genest, 1983; Ruiz Fernández, 1985; Spanos et al., 1984; Stam, Petrusic y Spanos, 1981; Stevenson, Kanfer y Higgins, 1984), estimulación eléctrica transcutánea y acupuntura (Ashton, Ebenezer, Golding y Thompson, 1984) o fármacos (Berntzen, Fors y Gotestam, 1985; García de Jalón et al., 1985; Posner, Telekes, Crowley, Phillipson y Peck, 1985). Sin embargo, no hay una normalización clara del procedimiento y se encuentran casi tantas variantes como investigadores. A pesar de ello, y a juzgar por el número de investigaciones que han utilizado el Cold Pressor Test como método de producción de dolor, podemos concluir que, junto con la estimulación eléctrica, es el método más utilizado en la investigación de dolor en laboratorio, particularmente en la evaluación de terapias psicológicas.

\section{TÉCNICAS DE REDUCCIÓN Y MANEJO DEL DOLOR ESTUDIA- DAS EN LABORATORIO}

Debemos comenzar este apartado señalando que el éxito de los procedimientos no significa necesariamente que la sensación de dolor desaparezca. Al igual que en la situación clínica, se considera que un tratamiento tiene éxito cuando la sensación de dolor se reduce, tras la intervención, del nivel intolerable al nivel tolerable. En términos de la teoría de la decisión sensorial, lo que ocurre frecuentemente es que la sensación permanece inalterada, pero deja de juzgarse como aversiva.

Al margen de los estudios tradicionales para la evaluación de la potencia analgésica de fármacos, hay una gran cantidad de investigaciones que ponen a prueba terapias de tipo psicológico -relajación, hipnosis, técnicas cognitivas y biorretroalimentación-, procedimientos alternativos como estimulación eléctrica transcutánea y acupuntura, o bien la conjunción de terapias farmacológicas y psicológicas. Otros trabajos de carácter más básico han intentado evaluar la influencia de diversos parámetros particulares de las cogniciones y expectativas del individuo previas a la estimulación aversiva, condiciones relevantes de la situación experimental, etc.- que interactúan dentro de la situación de laboratorio.

\subsection{Factores previos a la estimulación dolorosa}

Uno de estos trabajos lo constituye el ya clásico de Zimbardo, Cohen y Weisenberg, (1966) sobre disonancia cognitiva: se sometía a los 
sujetos a condiciones de alta o baja disonancia -según lo postulado por la teorla de Festinger (1957)- y después se les administraba una sesión de descargas eléctricas de igual intensidad a las que habían experimentado en una situación previa a la inducción de la situación de disonancia. De acuerdo a lo esperado, el grupo de sujetos sometidos a la condición de alta disonancia calificaron de intensidad moderada los niveles de descarga que en la sesión previa habían calificado de intensidad elevada. Por otra parte, un aspecto interesante de la investigación es el hallazgo de que el correlato fisiológico evaluado -resistencia electrodermal- que acompaña a la reacción de dolor era igual para estos individuos que para aquellos a los que realmente se administraba una descarga de intensidad moderada.

Otro aspecto de la situación cognitiva del sujeto previa a la estimulación dolorosa ha sido analizado por Hayes y Wolff (1984): si el sujeto cree que nadie conoce cuáles van a ser las estrategias de tipo psicológico que va a utilizar para hacer frente a la estimulación nociva, no se detectan efectos terapéuticos, mientras que sí los hay cuando el sujeto cree que otros conocen cuáles son esas estrategias. Dicho de otro modo, la existencia de un compromiso social puede modular la eficacia terapéutica de ciertos procedimientos de tipo psicológico.

Parece bastate claro, por otra parte, que tanto la predictibilidad como el control parecen reducir el impacto de los estímulos aversivos en los organismos tanto en animales como en humanos. Las investigaciones de laboratorio sugieren la posibilidad de que, bajo ciertas condiciones, control y predictibilidad pueden funcionar de modo intercambiable como señales de seguridad que reduzcan el impacto de los estímulos aversivos: cuando el comportamiento del sujeto no puede poner fina la estimulación aversiva, la predictibilidad puede reducir el impacto; cuando es imposible predecir realmente la ocurrencia y duración del estímulo aversivo, la falsa percepción del sujeto de que está en su mano poner fin a la estimulación, puede tener la misma función (Staub, Tursky y Schwartz, 1975). Sin embargo, Weisenberg, Wolf, Mittwoch, Mikulincer y Aviram (1985) han revisado las pruebas existentes respecto a la controlabilidad y predictibilidad y su capacidad reductora de la estimulación aversiva y han señalado la existencia de algunos estudios que permiten poner en duda dicha capacidad. Por otra parte, surge la cuestión sobre quién debe poseer, en la práctica clínica, el control y/o predictibilidad: el paciente o el terapeuta, ya que en muchas situaciones no todos los pacientes parecen dispuestos a aceptar la responsabilidad de ejercer el control sobre la situación y prefieren dejar dicha responsabilidad en manos del terapeuta. En el trabajo que acabamos de mencionar, Weisenberg y colaboradores intentan clarificar los efectos del control como variable mediadora en la reacción al dolor, en función de las variables 
predisposicionales "rasgo de ansiedad" y "autoeficacia" -constructo este último derivado de la teoría de Bandura (1977)-. Las conclusiones que parecen desprenderse de los resultados obtenidos reflejan la complejidad del problema: por una parte, la autoeficacia y el rasgo de ansiedad parecen ser buenos predictores de la reacción del individuo; por otra, el control que posee el experimentador redujo la reacción de dolor en algunos individuos pero no en otros; finalmente, añadir responsabilidad y control a un individuo altamente ansioso puede empeorar su reacción de dolor. En general, parece que el control que es percibido como inadecuado puede tener peores consecuencias que la ausencia de control.

Señalaremos también que el hecho de proporcionar información al sujeto sobre la forma de acción de un procedimiento parece aumentar su eficacia, al menos en el caso de la administración de fármacos (Dworkin et al. 1984).

De hecho, el efecto de las instrucciones que se dan previamente a los sujetos en los resultados que se obtienen debería entrar, lógicamente, dentro de este apartado, pero preferimos analizarlo con mayor detalle en el apartado 5. De cualquier modo, aparece con bastante claridad que si se proporciona a los sujetos instrucciones de tipo exigente, los sujetos soportan mejor la experiencia aversiva (Pancyr y Genest, 1983).

Finalmente, hay que señalar un grupo de procedimientos que actúan generando en el sujeto una analgesia previa a la estimulación aversiva por medio de la sugestión o la hipnosis (Hilgard et. al. 1974; Spanos et al. 1984; Stam et al. 1981) y que permiten disminuir las molestias producidas por dicha estimulación. Otra estrategia obtiene la analgesia a través del estrés (Willer, Dehen y Cambier, 1981). En ella, la capacidad analgésica se evalúa registrando los aumentos del umbral inferior de dolor del reflejo nociceptivo de flexión de una pierna: tras inducir estrés en los sujetos colocándoles en una situación en la que reciben descargas eléctricas impredecibles, se observa que la intensidad de estimulación necesaria para producir el reflejo aumenta considerablemente. La administración de naloxona disminuye esta capacidad analgésica por lo que se supone que en el proceso se halla involucrada una secreción aumentada de opiáceos endógenos debida a la propia situación de estrés.

\subsection{Procedimientos y técnicas ante la estimulación dolorosa}

La variedad de terapias, técnicas y procedimientos es considerable y en la presente revisión no podemos mencionarlos todos. Incluso dentro de procedimientos que tienen el mismo nombre se encuentran procesos y modos de actuación diferentes y, además, en muchas ocasiones, los términos empleados dan lugar a interpretaciones tan diversas que es difícil saber cuál de los elementos de la terapia fue efectivamente terapéu- 
tico y porqué. Por otra parte, la misma técnica se muestra eficaz en unas condiciones y no en otras, dependiendo del cómo y cuándo se aplique.

De forma general, podemos distinguir, entre los de carácter psicológico, dos tipos de procedimientos: los distractivos y los de reinterpretación de la situación.

La distracción es una de las estrategias más antiguas utilizadas contra el dolor y parece ser eficaz si el mismo no es muy intenso. Definiremos la distracción como la focalización de la atención del sujeto hacia estímulos alejados de las sensaciones o reacciones emocionales producidas por el estímulo nocivo. Así, cualquier estrategia cuyo propósito sea impedir el temor al estímulo doloroso o a sus efectos, será considerada una estrategia de distracción. La efectividad de estas técnicas se basa en la suposición de los límites de la capacidad atencional; es decir, las tareas de distracción que requieran mayor atención serán más reductoras de las molestias asociadas al dolor, siempre y cuando éste no sea de intensidad elevada.

En un artículo reciente de revisión del tema, McCaul y Malott (1984) concluyen que, a pesar de disponerse de datos experimentales favorables a la eficacia de estas técnicas, se necesitan más pruebas para poder afirmar su utilidad contra el dolor clínico: los factores que diferencian el dolor clínico y el de laboratorio pueden influir decisivamente en la viabilidad de la efectividad de las estrategias distractivas para afrontar el dolor de origen patológico.

Por nuestra parte, hemos hallado también algunos trabajos que apoyan la eficacia de las técnicas de distracción como procedimiento para afrontar el dolor (Ahles et al. 1983; Blitz y Dinnerstein, 1975) y sólo uno en el que estas técnicas no parecen ser eficaces (Ruiz Fernández, 1985).

El grupo de técnicas que denominamos "de reinterpretación de la situación" están encaminadas a que el individuo cambie sus cogniciones ante la estimulación dolorosa. Por ejemplo, en el procedimiento del Cold Pressor Test se utilizan estrategias como "piense que es un día de verano y en la sensación agradable que produce el agua fresca" y similares, las cuales parecen ser eficaces, al menos en esta situación de laboratorio (Ahles et al. 1983; Avia, 1980; Blitz y Dinnerstein, 1975; Geden, Beck, Hauge y Pohlman, 1984; Gilligan et al. 1984).

Otro tipo de estrategias utilizadas y que se han mostrado efectivas son la inoculación de estrés (De Blas y Labrador, 1984), convencer al sujeto de que es capaz de controlar las sensaciones que le produce la estimulación aversiva (Ruiz Fernández, 1985), fijar una meta razonable por ejemplo, resistir un cierto número de segundos- y hacer que el sujeto la consiga (Stevenson et al. 1984) y procedimientos de relajación 
cuya eficacia varía según el momento en que se lleva a cabo (Linton y Gotestam, 1983).

Entre los procedimientos no psicológicos que parecen revelarse eficaces, se encuentran la estimulación eléctrica transcutánea y la acupuntura (Ashton et al. 1984), fármacos como la codeína (García de Jalón et al. 1985) así como la producción de una estimulación dolorosa paralela aunque en este caso lo que se haga sea sustituir un dolor por otro de menor intensidad (Chen y Treede, 1985).

\section{PROBLEMAS DEL ESTUDIO DEL DOLOR EN EL LABORATORIO}

Ya hemos señalado las diferencias existentes entre el dolor experimental y el dolor clínico. Pero al margen de los componentes de duración, ansiedad y modo de producción, existe también el de la cualidad del dolor producido. En efecto, no es lo mismo un dolor artrítico, por ejemplo, que uno visceral o que el producido por una quemadura. Así pues, podríamos considerar un continuo dentro del dolor experimental al margen de la división que hemos hecho entre tónicos y fásicos- en el que se situarían los diferentes procedimientos en función de su semejanza con el dolor clínico. En un extremo del continuo -el que se hallaría más lejos del dolor clínico- situaríamos aquellos procedimientos que implican una estimulación de tipo más exteroceptivo y que afecta a los receptores cutáneos: métodos mecánicos de calor radiante y estimulación eléctrica, aunque esta última se encontraría un poco más próxima al dolor clínico. Aproximadamente en el centro de distribución situaríamos el Cold Pressor Test ya que la sensación de dolor no se localiza estrictamente en la zona cutánea. Por otra parte, la aplicación del Cold Pressor Test produce mayores puntuaciones en todas las escalas del McGill Pain Questionnaire que la aplicación de descargas eléctricas (Klepac, Dowling y Hauge, 1981). En la zona más proxima al dolor clínico colocaríamos los procedimientos químicos y la isquemia, ya que también estos últimos procedimientos -de tipo tónico- puntúan más alto que los de tipo fásico -estimulación eléctrica- en algunas escalas del McGill Pain Questionnaire (Chen y Treede, 1985). En particular, la isquemia suele ser considerada como el método más idóneo para reproducir en el laboratorio un dolor de tipo clínico en lo que se refiere a la cualidad. Como puede verse, cuanto más nos aproximamos a la dimensión de dolor clínico, más difícil es controlar los parámetros de medición y producción del mismo -intensidad de estímulo, tiempo de duración y aparición, etc.- aunque sea en situación de laboratorio. También puede observarse que aquellos procedimientos que más se asemejan al dolor clínico son los que hemos considerado como procedimientos tónicos. 
Pero al margen de los problemas que implica toda extrapolación del laboratorio a situaciones reales, las investigaciones experimentales tienen todas ellas aspectos y problemas intrínsecos que pueden influir en mayor o menor medida -a veces, con desconocimiento del propio investigador- en el proceso que se está analizando. En el caso del dolor experimental los problemas de procedimiento son múltiples; algunos de ellos tienen relación con problemas inherentes al método de producción del dolor: la descarga eléctrica genera más ansiedad que el Cold Pressor Test pero requiere un largo intervalo entre ensayos, lo que reduce su fiabilidad según algunos autores (Wolff, 1984); otros problemas se encuentran en el tipo de medida utilizada; así, no todos los sujetos son capaces de usar de forma adecuada una escala análoga visual (Kremer, Hampton e Ignelzi, 1981) y, en algunos casos, la capacidad de utilizar correctamente estas $u$ otras escalas puede verse mermada por la administración de los fármacos que se pretenden evaluar (Chapman et al 1985). Otro tipo de problemas que plantea el dolor experimental se deben al contexto de la situación y, de acuerdo con Avia (1979), destacaríamos que la escasa coherencia de los resultados obtenidos podría explicarse tanto por la diversidad de situaciones y estímulos aversivos utilizados como por la amplitud de la gama de variables dependientes que se han considerado como indicadores de reacciones al dolor. Desde otro punto de vista, algunos estudios utilizan medidas conductuales, y otros, en cambio, se sirven del informe verbal para determinar los umbrales.

Personalmente, destacaríamos la necesidad de establecer un nivel basal, previo a la intervención -lo cual algunos investigadores no hacencomo condición esencial para evaluar la eficacia de un procedimiento terapéutico. En el caso de existir dicha línea base, el intervalo entre ésta y la fase de prueba es otra condición que puede afectar a los resultados. Lo mismo puede decirse de la familiarización del sujeto con el estímulo al que va a frentarse o, simplemente, de la repetición de la medida (De Blas y Labrador, 1984); incluso puede existir una adaptación al estímulo doloroso, al menos en el Cold Pressor Test (Wolff, 1984). Otra problemática se suscita al decidir si se utiliza o no, la extremidad dominante del individuo (Murray y Hagan, 1975).

Son muy importantes, asimismo, las características de las demandas experimentales: los sujetos modifican su reacción ante la prueba según reciban instrucciones "exigentes" o "permisivas". Puesto que una inmensa mayoría de los trabajos conllevan fuertes demandas sobre los sujetos, en una gran parte de ellos resulta imposible discriminar si los resultados se han debido a la manipulación pretendida o, simplemente, a la coacción experimental (Pancyr y Genest, 1983). En función de si las instrucciones van dirigidas a modificar el umbral de retirada o el umbral 
inferior de dolor, se obtendrá un cambio en una $u$ otra de las magnitudes.

Por último, el contexto social de la situación en que tiene lugar la prueba influye tanto en las actividades de afrontamiento (coping) que realiza el sujeto y el grado de dolor que manifiesta, como en la interpretación que realiza de la situación (Spanos et al., 1984): así por ejemplo, si el sujeto observa la reacción al dolor de sujetos tolerantes o intolerantes al mismo, su propia reacción puede verse afectada en uno u otro sentido (Craig y Weiss, 1975).

\section{CONCLUSIONES}

No hay duda de la importancia de los estudios de laboratorio en la investigación del dolor, tanto en lo que se refiere a la determinación de los mecanismos neurológicos y bioquímicos como para evaluar el potencial analgésico de las terapias, sean estas farmacológicas o no farmacológicas. Por desgracia, las extrapolaciones que puedan hacerse de estos resultados a las situaciones clínicas no son siempre todo lo fiables y válidas que sería de desear, al margen de que la calidad de dolor de laboratorio se aproxime en mayor o menor medida a la del dolor clínico. Ya hemos señalado cuáles son las causas de esta heterogeneidad de resultados: por un lado, la gran diversidad de procedimientos utilizados $\mathrm{y}$, por otro, la escasa normalización de aplicación de estos procedimientos en los diversos estudios realizados hasta el momento. Por lo tanto, los resultados de las diversas investigaciones sobre factores que afectan al dolor y que hemos comentado en el apartado 4 deben ser consideradas teniendo en cuenta estas limitaciones e inconvenientes de la mayoría de trabajos existentes sobre dolor experimental.

Creemos que el problema actual no es tanto aproximar el dolor de laboratorio al dolor clínico -aunque esta pueda ser una línea de investigación relevante-, como conseguir que los estudios de laboratorio, dentro de las limitaciones inherentes a todas las investigaciones de este tipo, ofrezcan en sus resultados la mayor fiabilidad posible, permitiendo a diferentes investigadores una replicación destinada a conseguir conjuntos de resultados coherentes a partir de los cuales el terapeuta pueda llevar a cabo su trabajo, basándose en unos datos generados en una investigación básica correcta.

Está muy claro que en el dolor experimental no entran todos los componentes que pueden hallarse en el dolor clínico; ahora bien, si el investigador básico es capaz de suministrar al terapeuta una guía válida de la importancia y evolución de algunos de esos componentes en determinadas situaciones, qué duda cabe de que éste verá con mayor cla- 
ridad cómo enfrentarse con las situaciones clínicas. Los estudios de tipo estrictamente clínico complementarían la información que, en primera instancia, debe proporcionar la investigación básica.

En este sentido, sabemos que existe una jerarquía de fiabilidades retests para cuatro de los métodos más usados, que coloca la estimulación eléctrica cutánea como el más fiable, seguido por la radiación térmica, el algómetro de presión y el Cold Pressor Test (Wolff, 1978).

El procedimiento de la estimulación eléctrica de la piel arroja valores constantes en cualquier punto de ésta, no sufre variaciones en función de la hora del día, ni con el paso del tiempo y, por último, la familiaridad con el estímulo no hace variar el umbral inferior de dolor (Notermans, 1975). Los problemas del procedimiento isquémico ya han sido comentados $y$, sin duda, es el procedimiento menos fiable de los utilizados en laboratorio.

Partiendo de que disponemos ya de un procedimiento fásico de producción de dolor que se ha mostrado fiable, la estimulación eléctrica, creemos que debe potenciarse èl estudio de la fiabilidad de aquellos otros métodos que proporcionan un dolor tónico. Con los resultados que pueden obtenerse en el laboratorio sobre ambos tipos de dolor se ofrecerá un espectro de datos más rico que permitirá al clínico abordar mejor su tarea. Por supuesto, no es lo mismo juzgar una intensidad dolorosa que se presenta durante un breve tiempo que la misma intensidad durante un lapso de tiempo prolongado: la reacción ante el dolor variará considerablemente y lo que puede ser eficaz para el primer tipo de situaciones no necesariamente ha de serlo para el segundo. Debemos intentar obtener ambos tipos de información en situaciones de laboratorio en lo que refiere a la efectividad de la terapia antes de ofrecer alternativas al campo de la clínica. Más aún, en nuestra opinión los métodos fásicos son idóneos para la evaluación de analgésicos o procedimientos para aumentar el umbral inferior de dolor y los procedimientos tónicos deberían utilizarse en la evaluación de terapias contra un dolor que se mantiene durante largos períodos de tiempo y que no es sensible a los analgésicos; es decir, para evaluar estrategias fundamentalmente psicológicas.

El estudio sistemático del dolor, tanto experimental como clínico, se ha iniciado ya en los países de habla hispana (Avia, 1980; Bayés, 1983; Blasco, 1986; Blasco y Bayés, 1986; De Blas y Labrador, 1984; Penzo, 1986; Urraca y Labrador, 1986). Esperemos que estos trabajos iniciales no sólo no se interrumpan sino que pronto se multipliquen y consigan con su aportación a la comunidad científica ayudar a resolver este importante problema. 


\section{REFERENCIAS}

AHLES, T.A.; BLANCHARD, E.B. y LEVENTHAL, H. (1983): Cognitive control of pain: attention to the sensory aspects of the cold pressor stimulus. Cognitive Therapy and Research. 7 (2), 159-178.

ASHTON, H.; EBENEZER, I.; GOLDING, J.F. y THOMPSON, J.W. (1984): Effects of acupuncture and transcutaneous electrical nerve stimulation on cold induced pain in normal subjects. Joumal of Psychosomatic Research. 28 (4), 301-308.

AVIA, M.D. (1979):Los aspectos psicológicos en las reacciones a la estimulación aversiva: una revisión crítica. Análisis y Modificación de Conducta. 5 (9), 287-302.

AVIA, M.D. (1980): El control cognitivo del dolor: estrategias y técnicas. Revista de Psicologia General y Aplicada. 35(1), 99-114.

BANDURA, A. (1977): Self-efficacy: toward a theory of behavioral change. Psychological Review. 84, 191-215.

BAYÉS, R. (Ed.) (1983): Psicología i dolor. Quaderns de Psicologia. 7, 57-98.

BEECHER, H.K. (1975): Quantification of the subjective pain experience. En M. Weisenberg (Ed.) Pain: Clinical and experimental perspectives. 56-66. Saint-Louis, C.V. Mosby Company.

BERNTZEN, D., FORS, E. y GOTESTAM, K.G. (1985): Effect of an analgesic drug (paralgin forte) upon laboratory pain under different cognitive manipulations: an experimental study. Acta Neurologica Scandinavida. 72, 30-35.

BLASCO, T. (1986): Evaluación del dolor. Presentado para publicación.

BLASCO, T. y BAYÉS, R. (1986): Reliability of the Cold Pressor Test method. En preparación.

BLITZ, B. y DINNERSTEIN, A.J. (1975): Role of attentional focus in pain perception. Manipulation of response to noxius stimulation by instructions. En M. Wesenberg (Ed.). Pain: clinical and experimental perspectives. 177-180. St. Louis: C.V. Mosby Company.

BROMM, B. y MEIER, W. (1984): The intracutaneous stimulus: a new pain model for afgesimetric studies. Methodology and Findings in Experimental Clinical Pharmacology. 6(7), 405-410.

CHAPMAN, C.R., CASEY, K.L., DUBNER, R., FOLEY, K.M., GRACELY, R.H. y READING, A.E. (1985): Pain measurement: an overview. Pain. 22, 1-31.

CHEN, A.C.N. y TREEDE, R.D. (1985): The McGill Pain Questionnaire in the assesment of phasic and tonic experimental pain: behavioral evaluation of the "Pain Inhibiting" effect. Pain. 22, 67-69.

CHERY-CROZE, S. (1983): Relationship between noxious cold stimuli and the magnitude of pain sensation in man. Pain. 15, 265-269.

CRAING, K.D. y WEISS, S.M. (1975): Verbal reports of pain without noxious stimulation. En M. Weisenberg (Ed.). Pain: clinical and experimental perspectives. 203-206. St. Louis: C.V. Mosby Company.

DE BLAS, M.R. y LABRADOR, F.J. (1984): Eficacia de las técnicas de relajación, biofeedback de RPG e inoculación de estrés para modificar la percepción del dolor inducido experimentalmente. Análisis y Modificación de Conducta. 10(26), 513-532.

DWORKIN, S.F., CHEN, A.C.N., SCHUBERT, M.M. y CLARK, O.W. (1984): Cognitive modification of pain: information in combination with $\mathrm{N}_{2} \mathrm{O}$. Pain. 19, 339-351.

FESTINGER, L.A. (1957): A theony of cognitive dissonance. Row: Peterson.

FRUHSTORFER, H. y LINDBLOM, V. (1983): Vascular participation in deep cold pain. Pain. 17, 235-241.

GARCIA DE JALON, P.D., HARRISON, F.J.J., JOHNSON, K.I., KOZMA, C. y SCHNELLE, $K$ (1985): A modified cold stimulation technique for the evaluation of analgesic activity in human volunteers. Pain. 22, 183-189.

GEDEN, E., BECK, N., HAUGE, G. y POHLMAN, S. (1984): Self-report and psychophysiological effects of five pain-coping strategies. Nursing Research. 33(5), 260-265. 
GILLIGAN, R.M., ASCHER, L.M., WOLPER, J. y BOCHACHEVSKY, C. (1984): Comparison of theree cognitive strategies in altering pain behaviors on a cold pressor yask, Perceptual and Motor Skills. 59, 235-240.

HAYES, S.C. y WOLFF, M.R. (1984): Cues, consecuences and therapeutic talk: effects of social contex and coping statements on pain. Behaviour Research and Therapy. 22(4), 385-392.

HILGARD, E.R., RUCH, J.C., LANGE, A.F., LENOX, J.R., MORGAN, A.H. y SACHS, L.B. (1974): The psychophysics of cold pressor pain and its modification through hipnotic suggestion. American Joumal of Psychology. 87, 17-31.

KLEPAC, R.K., DOWLING, J. y HAUGE, G. (1981): Sensitivity of the McGill Pain Questionnaire to intensity and quality of laboratory pain. Pain. 10, 199-207.

KREMEN, E., HAMPTON, J. e IGNELZI, R.J. (1981): Measurement of pain: patient preference does not confound pain measurement. Pain. 10, 241-248.

LEWIS, J.W., CANNON, J.T. y LIEBESKIND, J.C. (1980): Opioid and nonoioid mechanisms of stress analgesia. Science. 208, 623-625.

LINTON, S.J. y GOTESTAM, K.G. (1983): Applied relaxation (coping) in control of laboratory pain: effects of signalled pain and instructions of when to relax. Psychological Reports. 53, 467-476.

MAIER, S.F., DRUGAN, R.C. y GRAU, J.W. (1982): Controllability, coping behavior and stress-induced analgesia in the rat. Pain. 12, 47-56.

McCAUL, K.D. y MALOTT, J.M. (1984): Distraction and coping with pain. Psychological Bulletin. 95(3), 516-533.

MIRÓ, M. (1983): Visió psicológica del dolor: dolor clínic i dolor experimental. Quaderns de Psicologia. 1, 59-64.

NOTERMANS, S.L.H. (1975): Measurement of the pain treshold determined by electrical stimulation and its clinical application. En M. Weisenberg (Ed.) .Pain: clinical and experimental perspectives. 72-87. St. Louis: C.V. Mosby Company.

MURRAY, F.S. y HAGAN, B.C. (1975): Pain treshold and tolerance of hands and feet. En M. Weisenberg (Ed.). Pain: clinical and experimental perspectives. 121-124. St.Louis: C.V. Mosby Company.

PANCYR, G. y GENEST, M. (1983): Demand characteristics in laboratory pain investigations: the cold pressor method. Póster presentado en el World Congress on Behavior Therapy en el 17 Annual AABT Convention, Washington.

PATKIN, M. (1975): Measurement of tenderness with the description a simple instrument. En M. Weisenberg (Ed.). Pain: clinical and experimental perspectives. 99-102. St.Louis. C.V. Mosby Company.

PENZO, W. (1986): Evaluación conductual del dolor crónico. Tesis doctoral inédita. Facultad de Medicina. Universidad de Barcelona.

POSNER, J., TELEKES, A., CROWLEY, D., PHILLIPSON, R. y PECK, A.W. (1985): Effects of an opiate on cold-induced pain and the CNS in healthy volunteers. Pain. 23, 73-82.

REVÉS, A. (1983): Revisió crítica i requisits dels métodes de producció experimental de dolor. Quaders de Psicologia. 1, 77-84.

RODRIGUEZ, A. (1983): Un nuevo instrumento para el estudio experimental del dolor: el Mecadol-1. Quaders de Psicologia. 1, 85-92.

RUIZ FERNANDEZ, M.A. (1985): Influencia de la inducción de control en las reacciones a la estimulación dolorosa. En prensa.

STAUB, E., TURKY, B. y SCHWARTZ, G.E. (1975): Self-control and predictability: their effects on reactions to aversive stimulation. En M. Weisenberg (Ed.). Pain: clinical and experimental perspectives. St. Louis: C.V. Mosby Company. 171-176.

SPANOS, N.P., HODGINS, D.C., STAM, H.J. y GWVNN, M. (1984): Suffering for science: the effects of implicit social demands on response to experimentally induced pain. Journal of Personality and Social Psychology. 46(5), 1162-1172.

STAM, H.J., PETRUSIC, W.M. y SPANOS, N.P. (1981): Magnitud scales for dold pressor pain. Perception and Psychophysics. 29(6), 612-617. 
STEVENSON, M.K., KANFER, F.H. y HIGGINS, J.M. (1984): Effects of goal specifity and time cues on pain tolerance. Cognitive Therapy and Research. 8(4), 415-426.

URRACA, S. y LABRADOR, F.J. (Eds.).(1986): Psicología del dolor. Jano. Medicina y Humanidades. $30,109-175$.

WEISENBERG, M. (1977): Pain and pain control. Psychological Bulletin. 84(5), 1008-1044. WEISENBERG, M., WOLFF, Y., MITTWOCH, T., MIKULINCER, M. y AVIRAM, D. (1985): Subject versus experimental control in the reaction to pain. Pain. 23, 187-200.

WILLER, J.C., DEHEN, H. y CAMBIER, J. (1981): Stress-induced analgesia in humans: endogenous opioids and noloxone-reversible depression of pain reflexes. Science. 212, $689-691$.

WOLFF, B.B. (1978): Behavioral measurement of humain pain. En R.A. Sternbach (Eds.) The psychology of pain. Nueva York: Raven Press.

WOLFF, B.B. (1983): Neurofisiologia i mesura del dolor. Ciéncia, 3, 156-160.

WOLFF, B.B. y JARVIK, M.E. (1975): Quantitative measures of deep somatic pain: futher studies with hipertonic saline. En M. Weisenberg (Ed.). Pain. Clinical and Experimental Perspectives. 88-98. St.Louis: C.V. Mosby Company.

WOOLF, C.J. (1984): Transcutaneous and implanted nerve stimulation. En P.D. Wall y R. Melzack (Eds.). Textbook of pain. 679-690. Londres: Churchill Livingstone.

ZIMBARDO, P.G., COHEN, A.R. y WEISENBERG, M. (1966): Control of pain motivation by cognitive dissonance. Science. 151, 217-219.

Toda correspondencia debe dirigirse a:

RAMON BAYÉS SOPENA

Laboratorio de Conducta

Area de Psicología Básica

Apartado 29

Universitat Autònoma de Barcelona

08193 Bellaterra (Barcelona) 